\title{
INSEPARABILITY AND STRONG HYPOTHESES FOR DISJOINT NP PAIRS
}

\author{
LANCE FORTNOW ${ }^{1}$ AND JACK H. LUTZ ${ }^{2}$ AND ELVIRA MAYORDOMO ${ }^{3}$ \\ ${ }^{1}$ Northwestern University, EECS Department, Evanston, Illinois, USA. \\ E-mail address: fortnow@eecs.northwestern.edu \\ ${ }^{2}$ Department of Computer Science, Iowa State University, Ames, IA 50011 USA. \\ E-mail address: lutz@cs.iastate.edu \\ ${ }^{2}$ Departamento de Informática e Ingeniería de Sistemas, Instituto de Investigación en Ingeniería de \\ Aragón, María de Luna 1, Universidad de Zaragoza, 50018 Zaragoza, SPAIN. \\ E-mail address: elvira\at\unizar.es
}

\begin{abstract}
This paper investigates the existence of inseparable disjoint pairs of NP languages and related strong hypotheses in computational complexity. Our main theorem says that, if NP does not have measure 0 in EXP, then there exist disjoint pairs of NP languages that are $\mathrm{P}$-inseparable, in fact $\operatorname{TIME}(2(\mathrm{n} \mathrm{k}))$-inseparable. We also relate these conditions to strong hypotheses concerning randomness and genericity of disjoint pairs.
\end{abstract}

\section{Introduction}

The main objective of complexity theory is to assess the intrinsic difficulties of naturally arising computational problems. It is often the case that a problem of interest can be formulated as a decision problem, or else associated with a decision problem of the same complexity, so much of complexity theory is focused on decision problems. Nevertheless, other types of problems also require investigation.

This paper concerns promise problems, a natural generalization of decision problems introduced by Even, Selman, and Yacobi [7]. A decision problem can be formulated as a set $A \subseteq\{0,1\}^{*}$, where a solution of this problem is an algorithm, circuit, or other device that decides $A$, i.e., tells whether or not an arbitrary input $x \in\{0,1\}^{*}$ is an element of $A$. In contrast, a promise problem is formulated as an ordered pair $(A, B)$ of disjoint sets $A, B \subseteq\{0,1\}^{*}$, where a solution is an algorithm or other device that decides any set $S \subseteq\{0,1\}^{*}$ such that $A \subseteq S$ and $B \cap S=\emptyset$. Such a set $S$ is called a separator of the disjoint pair $(A, B)$. Intuitively, if we are promised that every input will be an element of

1998 ACM Subject Classification: F.1.3.

Key words and phrases: Computational Complexity, Disjoint NP-pairs, Resource-Bounded Measure, Genericity.

Thanks: Fortnow's research supported in part by NSF grants CCF-0829754 and DMS-0652521. Lutz's research supported in part by National Science Foundation Grants 0344187, 0652569, and 0728806. Mayordomo's research supported in part by Spanish Government MICINN Project TIN2008-06582-C03-02.

$$
\text { Ste } \begin{aligned}
& \text { SYMPOSIUM } \\
& \text { ON THEORETICAL } \\
& \text { ASPECTS } \\
& \text { OF COMPUTER } \\
& \text { SCIENCE }
\end{aligned}
$$

27th Symposium on Theoretical Aspects of Computer Science, Nancy, 2010 Editors: Jean-Yves Marion, Thomas Schwentick

Leibniz International Proceedings in Informatics (LIPIcs), Schloss Dagstuhl - Leibniz-Zentrum für Informatik, Germany

Digital Object Identifier: 10.4230/LIPIcs.STACS.2010.2471

(C) L. Fortnow, J. H. Lutz, and E. Mayordomo

(C) Creative Commons Attribution-NoDerivs License 
$A \cup B$, then a separator of $(A, B)$ enables us to distinguish inputs in $A$ from inputs in $B$. Since each decision problem $A$ is clearly equivalent to the promise problem $\left(A, A^{c}\right)$, where $A^{c}=\{0,1\}^{*}-A$ is the complement of $A$, promise problems are, indeed, a generalization of decision problems.

A disjoint NP pair is a promise problem $(A, B)$ in which $A, B \in \mathrm{NP}$. Disjoint NP pairs were first investigated by Selman and others in connection with public key cryptosystems $[7,15,26,17]$. They were later investigated by Razborov [25] as a setting in which to prove the independence of complexity-theoretic conjectures from theories of bounded arithmetic. In this same paper, Razborov established a fundamental connection between disjoint NP pairs and propositional proof systems. Propositional proof systems had been used by Cook and Reckhow [6] to characterize the NP versus co-NP problem. Razborov [25] showed that each propositional proof system has associated with it a canonical disjoint NP pair and that important questions about propositional proof systems are thereby closely related to natural questions about disjoint NP pairs. This connection with propositional proof systems has motivated more recent work on disjoint NP pairs by Glaßer, Selman, Sengupta, and Zhang $[10,9,12,13]$. It is now known that the degree structure of propositional proof systems under the natural notion of proof simulation is identical to the degree structure of disjoint NP pairs under reducibility of separators [12]. Much of this recent work is surveyed in [11]. Goldreich [14] gives a recent survey of promise problems in general.

Our specific interest in this paper is the existence of disjoint NP pairs that are $\mathrm{P}$ inseparable, or even $\operatorname{TIME}\left(2^{n^{k}}\right)$-inseparable. As the terminology suggests, if $\mathcal{C}$ is a class of decision problems, then a disjoint pair is $\mathcal{C}$-inseparable if it has no separator in $\mathcal{C}$. The existence of P-inseparable disjoint NP pairs is a strong hypothesis in the sense that (1) it clearly implies $\mathrm{P} \neq \mathrm{NP}$, and (2) the converse implication is not known (and fails relative to some oracles [17]). It is clear that $\mathrm{P} \neq \mathrm{NP} \cap$ coNP implies the existence of P-inseparable disjoint NP pairs, and Grollmann and Selman [15] proved that $\mathrm{P} \neq \mathrm{UP}$ also implies the existence of P-inseparable disjoint NP pairs.

The hypothesis that NP is a non-measure 0 subset of EXP, written $\mu(\mathrm{NP} \mid \mathrm{EXP}) \neq 0$, is a strong hypothesis in the above sense. This hypothesis has been shown to have many consequences not known to follow from more traditional hypotheses such as $\mathrm{P} \neq \mathrm{NP}$ or the separation of the polynomial-time hierarchy into infinitely many levels. Each of these known consequences has resolved some pre-existing complexity-theoretic question in the way that agreed with the conjecture of most experts. This explanatory power of the $\mu(\mathrm{NP}$ EXP $) \neq 0$ hypothesis is discussed in the early survey papers $[23,2,24]$ and is further substantiated by more recent papers listed at [16] (and too numerous to discuss here). In several instances, the discovery that $\mu(\mathrm{NP} \mid \mathrm{EXP}) \neq 0$ implies some plausible conclusion has led to subsequent work deriving the same conclusion from some weaker hypothesis, thereby further illuminating the relationships among strong hypotheses.

Our main theorem states that, if NP does not have measure zero in EXP, then, for every positive integer $k$, there exist disjoint NP pairs that are $\operatorname{TIME}\left(2^{n^{k}}\right)$-inseparable. Such pairs are a fortiori P-inseparable, but the conclusion of our main theorem actually gives exponential lower bounds on the inseparability of some disjoint NP pairs. These are the lower bounds that most experts conjecture to be true, even though an unconditional proof of such bounds may be long in coming.

The proof of our main theorem combines known closure properties of NP with the randomness that the $\mu(\mathrm{NP} \mid \mathrm{EXP}) \neq 0$ hypothesis implies must be present in NP to give an explicit construction of a disjoint NP pair that is $\operatorname{TIME}\left(2^{n^{k}}\right)$-inseparable. (Technically, this 
is an overstatement. The last step of the "construction" is the removal of a finite set whose existence we prove, but which we do not construct.) The details are perhaps involved, but we preface the proof with an intuitive motivation for the approach.

We also investigate the relationships between the two strong hypotheses in our main theorem (i.e., its hypothesis and its conclusion) and strong hypotheses involving the existence of disjoint NP pairs with randomness and genericity properties. Roughly speaking (i.e., omitting quantitative parameters), we show that the existence of disjoint NP pairs that are random implies both the $\mu(\mathrm{NP} \mid \mathrm{EXP}) \neq 0$ hypothesis and the existence of disjoint NP pairs that are generic in the sense of Ambos-Spies, Fleischhack, and Huwig [1]. We also show that the existence of such generic pairs implies the existence of disjoint NP pairs that are $\operatorname{TIME}\left(2^{n^{k}}\right)$-inseparable. Taken together, these results give the four implications at the top of Figure 1. (The four implications at the bottom are well known.) We prove that three of these implications cannot be reversed by relativizable techniques, and we conjecture that this also holds for the remaining implication.

\section{Preliminaries}

We write $\mathbb{N}$ for the set of nonnegative integers and $\mathbb{Z}^{+}$for the set of (strictly) positive integers. The Boolean value of an assertion $\phi$ is $\llbracket \phi \rrbracket=$ if $\phi$ then 1 else 0 . All logarithms here are base- 2 .

We write $\lambda$ for the empty string, $|w|$ for the length of a string $w$, and $s_{0}, s_{1}, s_{2}, \ldots$ for the standard enumeration of $\{0,1\}^{*}$. The index of a string $x$ is the value $\operatorname{ind}(x) \in \mathbb{N}$ such that $s_{\text {ind }(x)}=x$. We write next $(x)$ for the string following $x$ in the standard enumeration, i.e., $\operatorname{next}\left(s_{n}\right)=s_{n+1}$. More generally, for $k \in \mathbb{N}$, we write next ${ }^{k}$ for the $k$-fold composition of next with itself, so that next ${ }^{k}\left(s_{n}\right)=s_{n+k}$.

A Boolean function is a function $f:\{0,1\}^{m} \rightarrow\{0,1\}$ for some $m \in \mathbb{N}$. The support of such a function $f$ is $\operatorname{supp}(f)=\left\{x \in\{0,1\}^{m} \mid f(x)=1\right\}$.

We write $w[i]$ for the $i^{\text {th }}$ symbol in a string $w$ and $w[i . . j]$ for the string consisting of the $i^{\text {th }}$ through $j^{\text {th }}$ symbols. The leftmost symbol of $w$ is $w[0]$, so that $w=w[0 . .|w|-1]$. For (infinite) sequences $S \in \Sigma^{\infty}$, the notations $S[i]$ and $S[i . . j]$ are defined similarly. A string $w \in \Sigma^{*}$ is a prefix of a string or sequence $x \in \Sigma^{*} \cup \Sigma^{\infty}$, and we write $w \sqsubseteq x$, if there is a string or sequence $y \in \Sigma^{*} \cup \Sigma^{\infty}$ such that $w y=x$. A language, or decision problem, is a set $A \subseteq\{0,1\}^{*}$. We identify each language $A$ with the sequence $A \in\{0,1\}^{\infty}$ defined by $A[n]=\llbracket s_{n} \in A \rrbracket$ for all $n \in \mathbb{N}$. If $A$ is a language, then expressions like $\lim _{w \rightarrow A} f(w)$ refer to prefixes $w \sqsubseteq A$, e.g., $\lim _{w \rightarrow A} f(w)=\lim _{n \rightarrow \infty} f(A[0 . . n-1])$.

A martingale is a function $d:\{0,1\}^{*} \rightarrow[0, \infty)$ satisfying

$$
d(w)=\frac{d(w 0)+d(w 1)}{2}
$$

for all $w \in\{0,1\}^{*}$. Intuitively, $d$ is a strategy for betting on the successive bits of a sequence $S \in\{0,1\}^{\infty}$ : The quantity $d(w)$ is the amount of money that the gambler using this strategy has after $|w|$ bets if $w \sqsubseteq S$. Condition (2.1) says that the payoffs are fair.

A martingale $d$ succeeds on a language $A \subseteq\{0,1\}^{*}$, and we write $A \in S^{\infty}[d]$, if $\lim \sup _{w \rightarrow A} d(w)=\infty$. If $t: \mathbb{N} \rightarrow \mathbb{N}$, then a martingale $d$ is (exactly) $t(n)$-computable if its values are rational and there is an algorithm that computes each $d(w)$ in $t(|w|)$ time. A 
martingale is p-computable if it is $n^{k}$-computable for some $k \in \mathbb{N}$, and it is $\mathrm{p}_{2}$-computable if it is $2^{(\log n)^{k}}$-computable for some $k \in \mathbb{N}$.

Definition 2.1. [22] Let $X$ be a set of languages, and let $R$ be a language.

(1) $X$ has p-measure 0 , and we write $\mu_{\mathrm{p}}(X)=0$, if there is a p-computable martingale $d$ such that $X \subseteq S^{\infty}[d]$. The condition $\mu_{\mathrm{p}_{2}}(X)=0$ is defined analogously.

(2) $X$ has measure 0 in EXP, and we write $\mu(X \mid \mathrm{EXP})=0$, if $\mu_{\mathrm{p}_{2}}(X \cap \mathrm{EXP})=0$.

(3) $R$ is p-random if $\mu_{\mathrm{p}}(\{R\}) \neq 0$, i.e., if there is no p-computable martingale that succeeds on $R$. Similarly, $R$ is $t(n)$-random if no $t(n)$ - computable martingale succeeds on $R$.

It is well known that these definitions impose a nontrivial measure structure on EXP [22]. For example, $\mu(\mathrm{EXP} \mid \mathrm{EXP}) \neq 0$.

We use the following fact in our arguments.

Lemma 2.2. [3, 18] The following five conditions are equivalent.

(1) $\mu(\mathrm{NP} \mid \mathrm{EXP}) \neq 0$.

(2) $\mu_{\mathrm{p}}(\mathrm{NP}) \neq 0$.

(3) $\mu_{\mathrm{p}_{2}}(\mathrm{NP}) \neq 0$.

(4) There exists a p-random language $R \in \mathrm{NP}$.

(5) For every $k \geq 2$, there exists an $2^{\log n^{k}}$-random language $R \in \mathrm{NP}$.

Finally, we note that $\mu(\mathrm{P} \mid \mathrm{EXP})=0[22]$, so $\mu(\mathrm{NP} \mid \mathrm{EXP}) \neq 0$ implies $\mathrm{P} \neq \mathrm{NP}$.

\section{Inseparable Disjoint NP Pairs and the Measure of NP}

This section presents our main theorem, which says that, if NP does not have measure 0 in EXP, then there are disjoint NP pairs that are P-inseparable. In fact, for each $k \in \mathbb{N}$, there is a disjoint NP pair that is $\operatorname{TIME}\left(2^{n^{k}}\right)$-inseparable.

It is convenient for our arguments to use a slight variant of the separability notion.

Definition 3.1. Let $(A, B)$ be a pair of (not necessarily disjoint) languages, and let $\mathcal{C}$ be a class of languages.

(1) A language $S \subseteq\{0,1\}^{*}$ almost separates $(A, B)$ if there is a finite set $D \subseteq\{0,1\}^{*}$ such that $S$ separates $(A-D, B-D)$.

(2) We say that $(A, B)$ is $\mathcal{C}$-almost separable if there is a language $S \in \mathcal{C}$ that almost separates $(A, B)$.

Observation 3.2. If a pair $(A, B)$ is not $\mathcal{C}$-almost separable, then $(A-D, B-D)$ is $\mathcal{C}$-inseparable for every finite set $D$.

Before proving our main theorem, we sketch the intuitive idea of the proof. We want to construct a disjoint NP pair $(A, B)$ that is $\mathrm{P}$-inseparable. Our hypothesis, that NP does not have measure 0 in EXP, implies that NP contains a language $R$ that is p-random. Since we are being intuitive, we ignore the subtleties of p-randomness and regard $R$ as a sequence of independent, fair coin tosses (with the $n^{\text {th }}$ toss heads iff $s_{n} \in R$ ) that just happens to be in NP. If we use these coins to randomly put strings in $A$ or $B$ but not both, we can count on the randomness to thwart any would-be separator in $\mathrm{P}$. 
The challenge here is that, if we are to deduce $A, B \in \mathrm{NP}$ from $R \in \mathrm{NP}$, we must make the conditions " $s_{n} \in A$ " and " $s_{n} \in B$ " depend on the coin tosses in a monotone way; i.e., adding a string to $R$ must not move a string out of $A$ or out of $B$.

This monotonicity restriction might at first seem to prevent us from ensuring that $A$ and $B$ are disjoint. However, this is not the case. Suppose that we decide membership of the $n^{\text {th }}$ string $s_{n}$ in $A$ and $B$ in the following manner. We toss $2 \log n$ independent coins. If the first $\log n$ tosses all come up heads, we put $s_{n}$ in $A$. If the second $\log n$ tosses all come up heads, we put $s_{n}$ in $B$. If our coin tosses are taken from $R$, which is in NP, then $A$ and $B$ will be in NP. Each string $s_{n}$ will be in $A$ with probability $\frac{1}{n}$, in $B$ with probability $\frac{1}{n}$, and in $A \cap B$ with probability $\frac{1}{n^{2}}$. Since $\sum_{n=1}^{\infty} \frac{1}{n}$ diverges and $\sum_{n=1}^{\infty} \frac{1}{n^{2}}$ converges, the first and second Borel-Cantelli lemmas tell us that $A$ and $B$ are infinite and $A \cap B$ is finite. Since $A \cap B$ is finite, we can subtract it from $A$ and $B$, leaving two disjoint NP languages that are, by the randomness of the construction, P-inseparable.

What prevents this intuitive argument from being a proof sketch is the fact that the language $R$ is not truly random, but only p-random. The proof that $A \cap B$ is finite thus becomes problematic. There is a resource-bounded extension of the first Borel-Cantelli lemma [22] that works for p-random sequences, but this extension requires the relevant sum of probabilities to be p-convergent, i.e., to converge much more quickly than $\sum_{n=1}^{\infty} \frac{1}{n^{2}}$.

Fortunately, in this particular instance, we can achieve our objective without p-convergence or the (classical or resource-bounded) Borel-Cantelli lemmas. We do this by modifying the above construction. Instead of putting the $n^{\text {th }}$ string into each language with probability $\frac{1}{n}$, we put each string $x$ into each of $A$ and $B$ with probability $2^{-|x|}$ so that $x$ is in $A \cap B$ with probability $2^{-2|x|}$. By the Cauchy condensation test, the relevant series have the same convergence behavior as those in our intuitive argument, but we can now replace slow approximations of tails of $\sum_{n=1}^{\infty} \frac{1}{n^{2}}$ with fast and exact computations of geometric series.

We now turn to the details.

Construction 3.3. (1) Define the functions $u, v:\{0,1\}^{*} \rightarrow\{0,1\}^{*}$ by the recursion

$$
\begin{aligned}
& u(\lambda)=\lambda, \\
& v(x)=\operatorname{next}^{|x|}(u(x)), \\
& u\left(\operatorname{next}(x)=\operatorname{next}^{|x|}(v(x)) .\right.
\end{aligned}
$$

(2) For each $x \in\{0,1\}^{*}$, define the intervals

$$
I_{x}=[u(x), v(x)), J_{x}=[v(x), u(n \operatorname{ext}(x))) .
$$

(3) For each $R \subseteq\{0,1\}^{*}$, define the languages

$$
\begin{aligned}
& A^{+}(R)=\left\{x \mid I_{x} \subseteq R\right\}, B^{+}(R)=\left\{x \mid J_{x} \subseteq R\right\}, \\
& A(R)=A^{+}(R)-B^{+}(R), B(R)=B^{+}(R)-A^{+}(R) .
\end{aligned}
$$

Note that each $\left|I_{x}\right|=\left|J_{x}\right|=|x|$. Also, $I_{\lambda}=J_{\lambda}=\emptyset\left(\right.$ so $\lambda \in A^{+}(R) \cap B^{+}(R)$ ), and

$$
I_{0}<J_{0}<I_{1}<J_{1}<I_{00}<J_{00}<I_{01}<\ldots,
$$

with these intervals covering all of $\{0,1\}^{*}$.

A routine witness argument gives the following.

Observation 3.4. $\quad(1)$ If $R \in \mathrm{NP}$, then $A^{+}(R), B^{+}(R) \in \mathrm{NP}$.

(2) If $R \in \mathrm{NP}$ and $\left|A^{+}(R) \cap B^{+}(R)\right|<\infty$, then $(A(R), B(R))$ is a disjoint NP pair.

We now prove two lemmas about Construction 3.3. 
Lemma 3.5. Let $k \in \mathbb{N}$. If $R \subseteq\{0,1\}^{*}$ is $2^{(\log n)^{k+2}}$-random, then $\left(A^{+}(R), B^{+}(R)\right)$ is not $\operatorname{TIME}\left(2^{n^{k}}\right)$-almost separable.

Lemma 3.6. If $R \subseteq\{0,1\}^{*}$ is $p$-random, then $\left|A^{+}(R) \cap B^{+}(R)\right|<\infty$.

We now have what we need to prove our main result.

Theorem 3.7. (main theorem) If NP does not have measure 0 in EXP, then, for every $k \in \mathbb{Z}^{+}$, there is a disjoint $N P$ pair that is $\operatorname{TIME}\left(2^{n^{k}}\right)$-inseparable, hence certainly $P$ inseparable.

Proof. Assume that $\mu(\mathrm{NP} \mid \mathrm{EXP}) \neq 0$, and let $k \in \mathbb{N}$. Then, by Lemma 2.2, there is a $2^{(\log n)^{k+2}}$-random language $R \in \mathrm{NP}$. By Lemma 3.5, the pair $\left(A^{+}(R), B^{+}(R)\right)$ is not $\operatorname{TIME}\left(2^{n^{k}}\right)$-almost separable. Since $R$ is certainly p-random, Lemma 3.6 tells us that $\left|A^{+}(R) \cap B^{+}(R)\right|<\infty$. It follows by Observation 3.4 that $(A(R), B(R))$ is a disjoint NP pair, and it follows by Observation 3.2 that $(A(R), B(R))$ is $\operatorname{TIME}\left(2^{n^{k}}\right)$-inseparable.

\section{Genericity and Measure of Disjoint NP Pairs}

In this section we introduce the natural notions of resource-bounded measure and genericity for disjoint pairs and relate them to the existence of P-inseparable pairs in NP. We compare the different strength hypothesis on the measure and genericity of NP and disjNP establishing all the relations in Figure 1.

Notation. Each disjoint pair $(A, B)$ will be coded as an infinite sequence $T \in\{-1,0,1\}^{\infty}$ defined by

$$
T[n]= \begin{cases}1 & \text { if } s_{n} \in A \\ -1 & \text { if } s_{n} \in B \\ 0 & \text { if } s_{n} \notin A \cup B\end{cases}
$$

We identify each disjoint pair with the corresponding sequence.

Resource-bounded genericity for disjoint pairs is the natural extension of the concept introduced for languages by Ambos-Spies, Fleischhack and Huwig [1].

Definition 4.1. A condition $C$ is a set $C \subseteq\{-1,0,1\}^{*}$. A $t(n)$-condition is a condition $C \in \operatorname{DTIME}(t(n))$. A condition $C$ is dense along a pair $(A, B)$ if there are infinitely many $n \in \mathbb{N}$ such that $(A, B)[0 . . n-1] i \in C$ for some $i \in\{-1,0,1\}$. A pair $(A, B)$ meets a condition $C$ if $(A, B)[0 . . n-1] \in C$ for some $n$. A pair $(A, B)$ is $t(n)$-generic if $(A, B)$ meets every $t(n)$-condition that is dense along $(A, B)$.

We first prove that generic pairs are inseparable.

Theorem 4.2. Every $t(\log n)$-generic disjoint pair is $\operatorname{TIME}(t(n))$-inseparable.

We can now relate genericity in disjNP and inseparable pairs as follows.

Corollary 4.3. If disjNP contains a $2^{(\log n)^{k}}$-generic pair for every $k \in \mathbb{N}$, then disjNP contains a TIME $\left(2^{n^{k}}\right)$-inseparable pair for every $k \in \mathbb{N}$.

Resource-bounded measure on classes of disjoint pairs is the natural extension of the concept introduced for languages by Lutz [22], and is defined by using martingales on a three-symbol alphabet as follows. 
Definition 4.4. (1) A pair martingale is a function $d:\{-1,0,1\}^{*} \rightarrow[0, \infty)$ such that for every $w \in\{-1,0,1\}^{*}$

$$
d(w)=\frac{1}{4} d(w 0)+\frac{3}{8} d(w 1)+\frac{3}{8} d(w(-1)) .
$$

(2) A pair martingale $d$ succeeds on a pair $(A, B)$ if $\lim _{\sup } \rightarrow(A, B) d(w)=\infty$.

(3) A pair martingale $d$ succeeds on a class of pairs $X \subseteq\{-1,0,1\}^{\infty}$ if it succeeds on each $(A, B) \in X$.

Our intuitive rationale for the coefficients in part 1 of this definition is the following. We toss one fair coin to decide whether $s_{|w|} \in A$ and another to decide whether $s_{|w|} \in B$. If both coins come up heads, we toss a third coin to break the tie. The reader may feel that some other coefficients, such as $\frac{1}{3}, \frac{1}{3}, \frac{1}{3}$ are more natural here. Fortunately, a routine extension of the main theorem of [5] shows that the value of $\mu(\operatorname{disjNP} \mid \operatorname{disjEXP})$ will be the same for any choice of three positive coefficients summing to 1 .

When restricting martingales to those computable within a certain resource bound, we obtain a resource-bounded measure that is useful within a complexity class. Here we are interested in the class of disjoint EXP pairs, disjEXP.

Definition 4.5. (1) Let $\mathrm{p}_{2}$ be the class of functions that can be computed in time $2^{(\log n)^{O(1)}}$.

(2) A class of pairs $X \subseteq\{-1,0,1\}^{\infty}$ has $\mathrm{p}_{2}$-measure 0 if there is a martingale $d \in \mathrm{p}_{2}$ that succeeds on $X$.

(3) $X \subseteq\{-1,0,1\}^{\infty}$ has $\mathrm{p}_{2}$-measure 1 if $X^{c}$ has $\mathrm{p}_{2}$-measure 0 .

(4) A class of pairs $X \subseteq\{-1,0,1\}^{\infty}$ has measure 0 in disjEXP, denoted $\mu(X \mid \operatorname{disjEXP})=$ 0 , if $X \cap$ disjEXP has $\mathrm{p}_{2}$-measure 0 .

(5) $X \subseteq\{-1,0,1\}^{\infty}$ has measure 1 in disjEXP if $X^{c}$ has measure 0 in disjEXP.

It is easy to verify that $\mathrm{p}_{2}$-measure is nontrivial on disjEXP (as proven for languages in $[22])$.

In the following we consider the hypothesis that disjNP does not have measure 0 in disjEXP (written $\mu(\operatorname{disjNP} \mid \operatorname{disjEXP}) \neq 0$ ). We start by proving that this hypothesis is at least as strong as the well studied $\mu(\mathrm{NP} \mid \mathrm{EXP}) \neq 0$ hypothesis.

Theorem 4.6. If $\mu(\operatorname{disjNP} \mid \operatorname{disjEXP}) \neq 0$ then $\mu(\mathrm{NP} \mid \mathrm{EXP}) \neq 0$.

We finish by relating measure and genericity for disjoint pairs.

Theorem 4.7. If $\mu(\operatorname{disjNP} \mid \operatorname{disjEXP}) \neq 0$, then disjNP contains a $2^{(\log n)^{k}}$-generic pair for every $k \in \mathbb{N}$.

\section{Oracle Results}

All the techniques in this and related papers relativize, that is they hold when all machines involved have access to the same oracle $A$. In this section we give relativized worlds where the converses of most of the results in this paper, as expressed in Figure 1, do not hold. Since the implications trivially all hold in any relativized world where $\mathrm{P}=\mathrm{NP}[4]$, one cannot use relativizable techniques to settle these converses.

We'll work our way from the bottom up of Figure 1. 


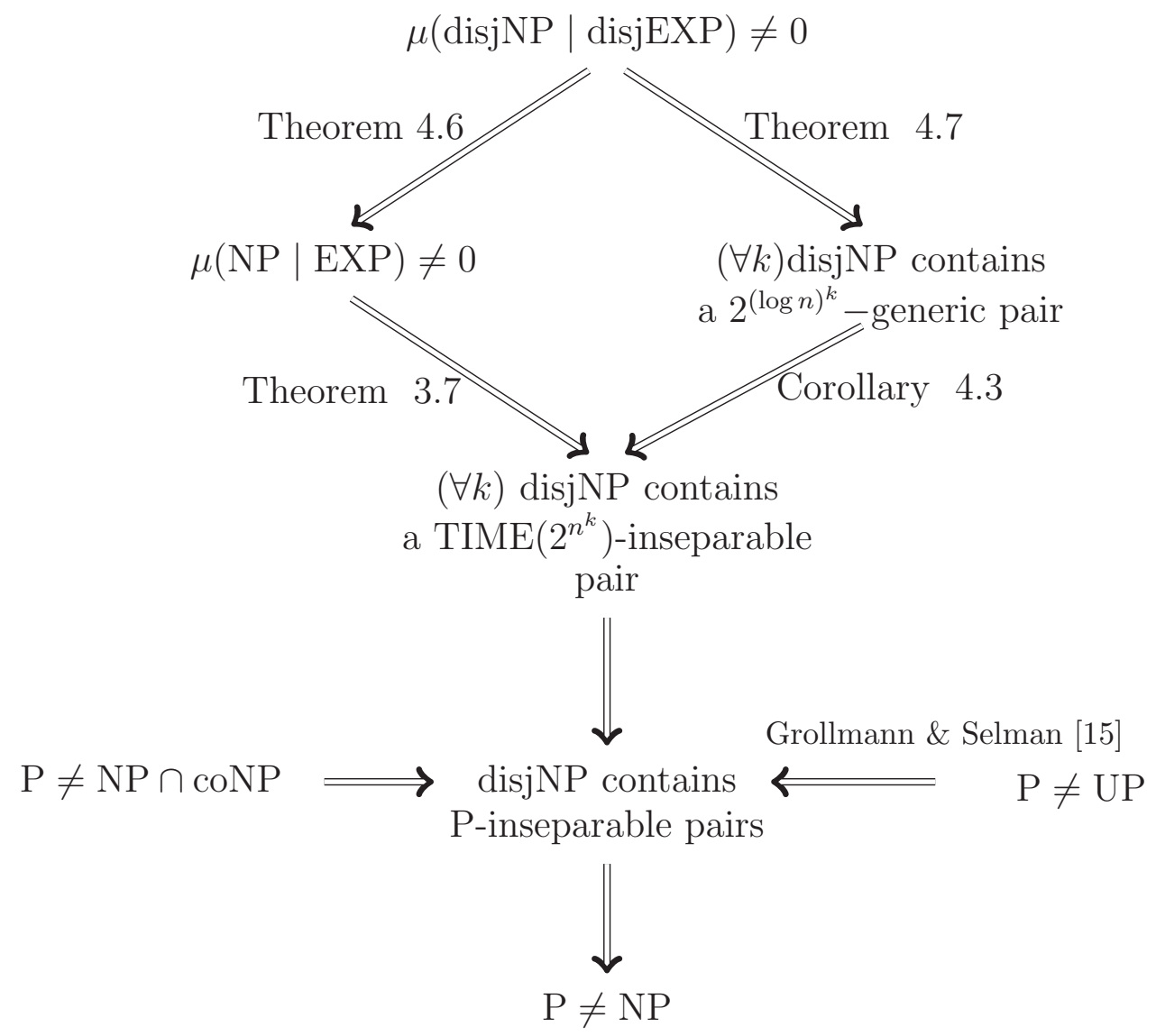

Figure 1: Relations among some strong hypotheses.

Theorem 5.1 (Homer-Selman [17], Fortnow-Rogers [8]). There exists oracles $A$ and $B$ such that

- $\mathrm{P}^{A} \neq \mathrm{NP}^{A}$ and disjNP ${ }^{A}$ does not contain $\mathrm{P}^{A}$-inseparable pairs.

- $\mathrm{P}^{B}=\mathrm{NP}^{B} \cap \operatorname{coNP}^{B}=\mathrm{UP}^{B}$ and disjNP $\mathrm{N}^{B}$ does contain $\mathrm{P}^{B}$-inseparable pairs.

Theorem 5.2. There exists an oracle $C$ such that $\mathrm{P}^{C} \neq \mathrm{UP}^{C}$ but $\mathrm{NP}^{C}$ is contained in $\operatorname{TIME}^{C}\left(n^{O(\log n)}\right)$. In particular this means that relative to $C$, disjNP contains $\mathrm{P}$-inseparable pairs but there is a $k$ (and in fact any real $k>0$ ) such that $\operatorname{disjNP}$ has no $\operatorname{TIME}\left(2^{n^{k}}\right)$ inseparable pairs.

Theorem 5.3. There exists a relativized world D, relative to which for all $k$, disjNP contains a $\operatorname{TIME}\left(2^{n^{k}}\right)$-inseparable pair but $\mu(\mathrm{NP} \mid \mathrm{EXP})=0$ and disjNP does not contain a $2^{(\log n)^{k}}$-generic pair.

Theorem 5.4. There exists an oracle $E$ relative to which for all $k$, disjNP contains a $2^{(\log n)^{k}}$-generic pair but $\mu(\operatorname{disj} \mathrm{NP} \mid \operatorname{disjEXP})=0$.

Conjecture 5.5. There exists an oracle $H$ relative to which $\mu(\mathrm{NP} \mid \mathrm{EXP}) \neq 0$ but $\mu(\operatorname{disjNP} \mid \operatorname{disjEXP})=0$. 
Let $K$ be a PSPACE-compete set, $R$ be a "random" oracle and let

$$
H=K \oplus R=\{\langle 0, x\rangle \mid x \in K\} \cup\{\langle 1, y\rangle \mid y \in R\} .
$$

Kautz and Miltersen show in $[20]$ that relative to $H, \mu(\mathrm{NP} \mid \mathrm{EXP}) \neq 0$. Kahn, Saks and Smyth [19] combined with unpublished work of Impagliazzo and Rudich show that relative to $H$ there is a polynomial-time algorithm that solves languages in $\mathrm{NP} \cap$ coNP on average for infinitely-many lengths which would imply $\mu(\mathrm{NP} \cap \operatorname{coNP} \mid \mathrm{EXP})=0$ relative to $H$. We conjecture that one can modify this proof to show $\mu\left(\operatorname{disjNP}{ }^{H} \mid \operatorname{disj}_{E_{X P}}\right)=0$.

\section{References}

[1] K. Ambos-Spies, H. Fleischhack, and H. Huwig. Diagonalizations over polynomial time computable sets. Theoretical Computer Science, 51:177-204, 1987.

[2] K. Ambos-Spies and E. Mayordomo. Resource-bounded measure and randomness. In A. Sorbi, editor, Complexity, Logic and Recursion Theory, Lecture Notes in Pure and Applied Mathematics, pages 1-47. Marcel Dekker, New York, N.Y., 1997.

[3] K. Ambos-Spies, S. A. Terwijn, and X. Zheng. Resource bounded randomness and weakly complete problems. Theoretical Computer Science, 172:195-207, 1997.

[4] T. Baker, J. Gill, and R. Solovay. Relativizations of the P =? NP question. SIAM Journal on Computing, 4:431-442, 1975.

[5] J.M. Breutzmann and J.H. Lutz. Equivalence of measures of complexity classes. SIAM Journal on Computing, 29(1):302-326, 1999.

[6] S. Cook and R. Reckhow. The relative efficiency of propositional proof systems. Journal of Symbolic Logic, 44:36-50, 1979.

[7] S. Even, A. L. Selman, and Y. Yacobi. The complexity of promise problems with applications to publickey cryptography. Information and Control, 61(2):159-173, 1984.

[8] L. Fortnow and J. Rogers. Separability and one-way functions. Computational Complexity, 11:137-157, 2002.

[9] C. Glaßer, A. L. Selman, and S. Sengupta. Reductions between disjoint NP-pairs. Information and Computation, 200:247-267, 2005.

[10] C. Glaßer, A. L. Selman, S. Sengupta, and L. Zhang. Disjoint NP-pairs. SIAM Journal on Computing, 33:1369-1416, 2004.

[11] C. Glaßer, A. L. Selman, and L. Zhang. Survey of disjoint NP-pairs and relations to propositional proof systems. In Theoretical Computer Science: Essays in Memory of Shimon Even, pages 241-253. Springer, 2006.

[12] C. Glaßer, A. L. Selman, and L. Zhang. Canonical disjoint NP-pairs of propositional proof systems. Theoretical Computer Science, 370:60-73, 2007.

[13] C. Glaßer, A. L. Selman, and L. Zhang. The informational content of canonical disjoint NP-pairs. In COCOON, LNCS, pages 307-317. Springer, 2007.

[14] O. Goldreich. On promise problems: A survey. In Theoretical Computer Science: Essays in Memory of Shimon Even, pages 254-290. Springer, 2006.

[15] J. Grollmann and A. Selman. Complexity measures for public-key cryptosystems. SIAM Journal on Computing, 11:309-335, 1988.

[16] J.M. Hitchcock. Resource-bounded measure bibliography. http://www.cs.uwyo.edu/ jhitchco/bib/rbm.shtml.

[17] S. Homer and A. L. Selman. Oracles for structural properties: The isomorphism problem and public-key cryptography. Journal of Computer and System Sciences, 44:287-301, 1992.

[18] D.W. Juedes and J.H. Lutz. Weak completeness in E and $E_{2}, 1995$.

[19] J. Kahn, M. E. Saks, and C. D. Smyth:. A dual version of reimer's inequality and a proof of rudich's conjecture. In Proceedings of the 15th Annual IEEE Conference on Computational Complexity, pages 98-103, 2000.

[20] S. M. Kautz and P. B. Miltersen. Relative to a random oracle, NP is not small. Journal of Computer and System Sciences, 53:235-250, 1996.

[21] A.K. Lorentz and J.H. Lutz. Genericity and randomness over feasible probability measures. Theoretical Computer Science, 207(1):245-259, 1998. 
[22] J. H. Lutz. Almost everywhere high nonuniform complexity. Journal of Computer and System Sciences, 44(2):220-258, 1992.

[23] J. H. Lutz. The quantitative structure of exponential time. In L. A. Hemaspaandra and A. L. Selman, editors, Complexity Theory Retrospective II, pages 225-254. Springer-Verlag, 1997.

[24] J. H. Lutz and E. Mayordomo. Twelve problems in resource-bounded measure. In G. Păun, G. Rozenberg, and A. Salomaa, editors, Current Trends in Theoretical Computer Science, entering the 21st century, pages 83-101. World Scientific Publishing, 2001.

[25] A. Razborov. On provably disjoint NP pairs. Technical Report 94-006, ECCC, 1994.

[26] A.L. Selman. Complexity issues in cryptography. In Computational complexity theory (Atlanta, GA, 1988), volume 38 of Proc. Sympos. Appl. Math., pages 92-107. Amer. Math. Soc., 1989. 